\title{
Impervious Coronary No-Reflow: A Case Study
}

\section{Karimianpour A and Maran A*}

Department of Cardiovascular Diseases, Heart \& Vascular Institute, Medical University of South Carolina, USA

\begin{abstract}
Coronary no-reflow phenomenon is a disorder of the microvasculature that results in poor myocardial perfusion in patients with acute myocardial infarction receiving percutaneous coronary intervention. It typically manifests as TIMI 1-2 flow, high TIMI frame counts, and abnormal myocardial blush. The etiology of this condition is multifactorial and so is its treatment. It is encountered in approximately one-third of patients and is therefore of clinical relevance. In this case report, we describe a patient scenario with this condition in which the standard therapy was provided but failed to achieve normal perfusion despite repeat attempts. We also discuss different pharmacological therapies that compose the standard therapy and other adjunctive remedies that have been proven to be beneficial.
\end{abstract}

Keywords: No-reflow; Coronary intervention; Myocardial perfusion; Microvascular obstruction

\section{Introduction}

Coronary no-reflow phenomenon is a disorder of the microvascular bed that results in poor perfusion of the myocardium despite revascularization of the epicardial coronary artery during an acute myocardial infarction [1,2]. After percutaneous coronary intervention (PCI), epicardial coronary filling is assessed using the Thrombolysis In Myocardial Infarction risk score (TIMI) flow grading system, TIMI frame count, and myocardial blush grading $[2,3]$. Coronary slow-reflow or no-reflow is diagnosed when TIMI flow grades of 1 or 2, high TIMI frame count, or abnormal myocardial blush is observed immediately post PCI [2]. The phenomenon occurs in approximately $30 \%$ of patients receiving PCI for acute myocardial infarction [4,5]. The proposed etiology of coronary no-reflow phenomenon is multifactorial and includes distal embolization of thrombus fragments during PCI, inflammation of the endothelium leading to microvascular dysfunction, reperfusion injury, and vasospasm [6-8]. Therefore, its treatment is founded in the proposed pathophysiology. In this case report we discuss a patient scenario in which coronary no-reflow phenomenon was observed and managed in the cardiac catheterization laboratory.

\section{Patient Scenario}

A 42-year-old otherwise healthy male presents with symptoms reminiscent of typical angina. He describes the pain as a substernal heavy pressure that radiates to his left arm, associated with diaphoresis, nausea, and tingling in his fingers. Upon driving himself to the chest pain center of our university hospital, he is noted to be hypertensive and with no ischemic changes on electrocardiography. Initial serum cardiac biomarkers returned negative. His pain was minimally relieved with sublingual nitroglycerin tablets.

Upon solicitation of a more detailed history, it became apparent that his father had suffered from an acute myocardial infarction at the age of 50. The patient himself was an active tobacco and marijuana user, and although he had not been diagnosed with any medical conditions prior to this, he was noted to be hyperglycemic on admission.

As part of the work-up in the chest pain center, a computed tomography angiography of the heart suggested a total occlusion of the left circumflex artery just distal to the first obtuse marginal branch with hypo attenuation of the subtended myocardium concerning for acute myocardial infarction (Figure 1). Shortly after the result of this scan, his serum cardiac biomarkers began to rise. At this point, the decision was made to transition the patient to the cardiac catheterization laboratory for urgent catheterization.

Coronary angiography revealed an occluded large caliber atrioventricular circumflex artery (Figure 2). Due to the total occlusion, aspiration thrombectomy was performed to assist in the passage of the stent and balloon catheters across the lesion. Intravascular ultrasound demonstrated an aneurysmal segment within the area of stenosis. The lesion was then treated with pre-dilation followed by deployment of a drug-eluting stent, and post-dilation of the distal vessel utilizing low pressure.

At this point, coronary slow-reflow was observed even though the patient was chest pain free. Repeat doses of intracoronary nicardipine and nitroglycerin were administered in conjunction with repeat balloon angioplasty. Despite all efforts, the final angiogram demonstrated TIMI II flow at best (Video 1).

\section{Discussion}

The development of coronary no-reflow phenomenon has been associated with several pre-procedural and intraoperative risk factors. Age, tobacco use, door-to-balloon time, initial perfusion defect, previous myocardial infarction, serum creatinine, C-reactive protein levels, left ventricular ejection fraction and Killip classification score have been associated with this condition [9]. Our patient although very young, did possess these certain risk factors including smoking, and a delayed door-to-balloon time as his electrocardiogram was normal on presentation and coronary catheterization was pursued urgently rather than emergently. Circumflex coronary artery occlusions tend to be silent and treatment is often delayed because of absence of electrocardiographic changes.

*Corresponding author: Anbukarasi Maran, Department of Cardiovascular Diseases, Heart \& Vascular Institute, Medical University of South Carolina, 171 Ashley Ave, Charleston, SC 29425, USA, Tel: 843-876-4788/843-792-1952 E-mail: maran@musc.edu

Received December 07, 2017; Accepted December 22, 2017; Published December 29,2017

Citation: Karimianpour A, Maran A (2017) Impervious Coronary No-Reflow: A Case Study. Cardiovasc Pharm Open Access 6: 229. doi: 10.4172/2329-6607.1000229

Copyright: ( 2017 Karimianpour A, et al. This is an open-access article distributed under the terms of the Creative Commons Attribution License, which permits unrestricted use, distribution, and reproduction in any medium, provided the original author and source are credited. 


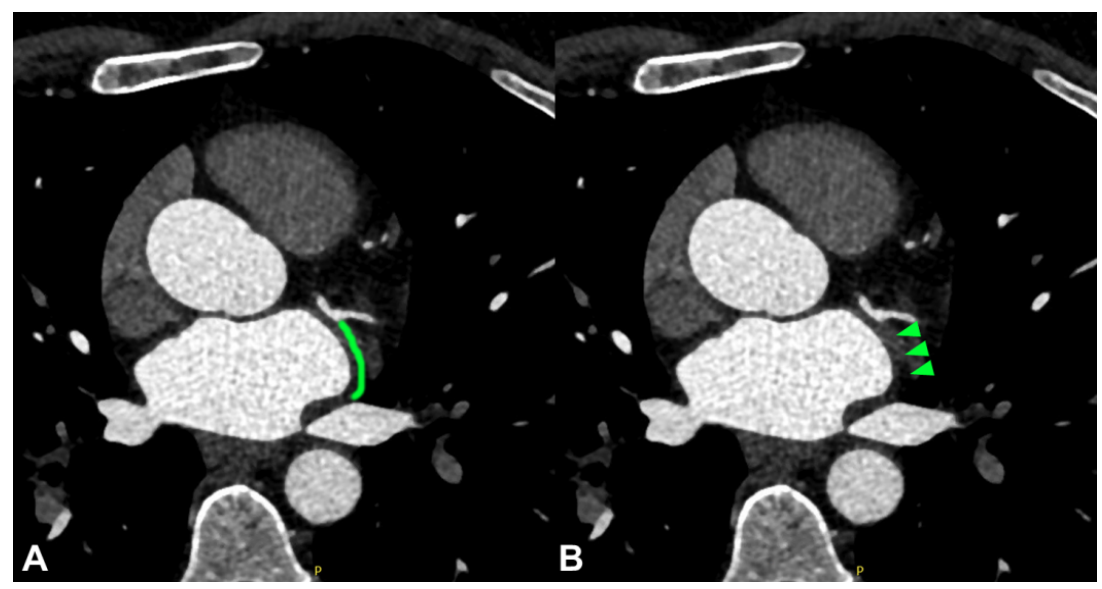

Figure 1: Computed tomography angiography of the heart. Panel A depicts the normal course of the left circumflex coronary artery in green. Panel B depicts the missing left circumflex coronary artery due to a total occlusion as shown by the green arrow-heads.

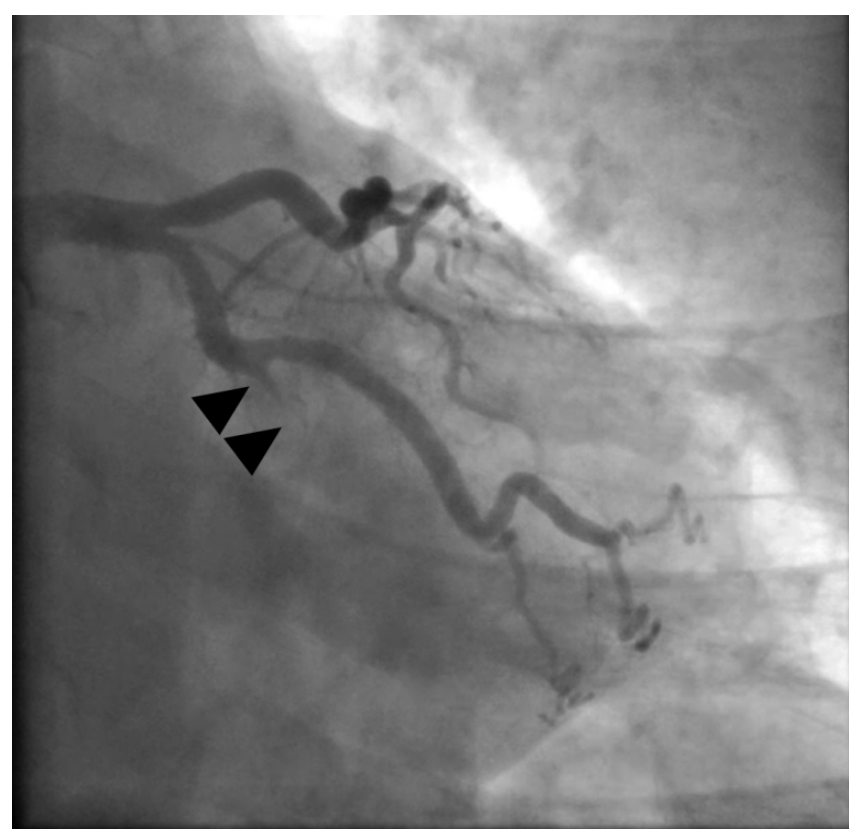

Figure 2: Coronary angiography depicting the total occlusion of the circumflex coronary artery as shown by the black arrow-heads.

Additionally, acute hyperglycemia (regardless of the diagnosis of diabetes mellitus), and hypertension are strongly associated with the development of coronary no-reflow [10-12]. Although our patient's serum glucose levels were not as high as the patient population studied by Iwakura and colleagues, it may have played a role.

Once coronary no-reflow phenomenon is observed in the cardiac catheterization lab, certain pharmacologic therapies have been proposed and shown to be of benefit, as discussed below.

\section{Standard pharmacologic therapies}

The pharmacologic treatment of coronary no-reflow revolves around the multifactorial pathophysiology of the condition. Typically, multiple agents are used in conjunction with non-pharmacologic techniques in the cardiac catheterization laboratory.

Adenosine has been studied extensively for its vasodilatory effect when injected into the coronary arteries, and also its properties

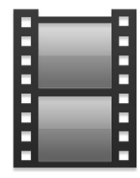

Video 1: Coronary angiography depicting the final result of percutaneous coronary intervention with coronary slow-reflow.

that result in inhibition of platelet aggregation and thromboemboli formation [13-15]. However, its short half-life, and potential side effect of atrioventricular nodal blockade and hypotension may limit its use and benefit [16]. Nonetheless, it remains as an available therapy for coronary no-reflow phenomenon.

Sodium nitroprusside and other nitrates alike, when given intracoronary, results in a greater and more prolonged vasodilation and myocardial hyperemia [17]. Several meta analyses have confirmed the benefit of intracoronary sodium nitroprusside for the prevention and treatment of coronary no re-flow phenomenon $[18,19]$.

Nondihydropyridine calcium channel blockers have been studied and shown to be beneficial in treating this condition [20]. However, nicardipine, a dihydropyridine calcium channel blocker, has been shown to be very successful in treatment of coronary no-reflow given its safer profile in regards to negative ionotropy which limit the usage and frequent dosing of its counterpart nondihydropyridine calcium channel blockers diltiazem and verapamil [21]. 


\section{Other pharmacologic therapies}

In patients who are on chronic aspirin therapy prior to an acute myocardial infarction and PCI, less coronary no-reflow has been observed [22]. Similarly, anti-platelet therapy with P2Y12 inhibitors has also been associated with mitigation of this condition given its mechanisms responsible for inhibition of platelet aggregation [23]. Furthermore, glycoprotein IIb/IIIa inhibitors, such as abciximab, have been used successfully to improve microcirculation by reducing thrombus burden and lessening distal embolization of thrombus fragments during PCI [24].

Agents such as cyclosporine-A, FX06 (a human fibrin peptide), pexelizumab, and dabigatran have also been studied in this phenomenon with variable results [25-28]. Unfortunately, these therapies as compared to the aforementioned standard therapies have not received much traction in practice.

Liraglutide has been recently studied in human models and noted to reduce incidence of coronary no-reflow, an effect that has been attributed to its ability in reducing inflammation and endothelial dysfunction by regulating peri-procedural serum glucose levels [29]. Endogenous erythropoietin levels have also been associated with improved microvascular function through reduction in inflammation and apoptosis, and increases in nitric oxide within the vascular bed [30]. Though both are innovative in their approach and application, further research and randomized controlled trials are needed to study their benefit in human subjects.

Finally, nicorandil, a mitochondrial potassium channel modulator and nicotamide nitrate, has proven its great efficacy in multiple international studies for the treatment of coronary no-reflow with associated improvement in left ventricular ejection fraction and filling pressures over time [31,32]. Nicorandil is currently not available in the United States.

\section{Conclusion}

In this case study, we describe a patient scenario of silent myocardial infarction with delayed treatment for acute occlusion of circumflex artery. Despite reperfusion, the patient experienced coronary slowreflow phenomenon. With multiple Balloon Angioplasties and the use of intracoronary nicardipine and nitroglycerin, the slow-reflow phenomenon improved from TIMI 0 to TIMI II flow. As discussed above, several modifiable and non-modifiable factors may have resulted in the development of this condition. The current standard of care falls short in identifying patients who are at high risk for coronary reflow and the treatment options are limited. Given the high incidence of this phenomenon, further investigation into the pathophysiology and treatment of this condition is needed.

\section{References}

1. Bouleti C, Mewton N, Germain S (2015) The no-reflow phenomenon: state of the art. Arch Cardiovasc Dis 108: 661-674.

2. Rezkalla SH, Stankowski RV (2017) Management of No-Reflow Phenomenon in the Catheterization Laboratory. J Am Coll Cardiol 10: 215-223.

3. Gibson CM, Murphy SA, Rizzo MJ (1999) Thrombolysis in Myocardial Infarction (TIMI) Study Group. Relationship between TIMI frame count and clinical outcomes after thrombolytic administration. Circulation 99: 1945-1950.

4. Morishima I (1995) Clinical significance of no-reflow phenomenon observed on angiography after successful treatment of acute myocardial infarction with percutaneous transluminal coronary angioplasty. Am Heart J 130: 239-243.

5. Rezkalla SH, Dharmashankar KC, Abdalrahman IB, Kloner RA (2010) Noreflow phenomenon following percutaneous coronary intervention for acute myocardial infarction: incidence, outcome, and effect of pharmacologic therapy. J Interv Cardiol 23: 429-436.
6. Schofer J, Montz R, Mathey DG (1985) Scintigraphic evidence of the "no reflow" phenomenon in human beings after coronary thrombolysis. J Am Coll Cardiol 5: 593-598.

7. Kloner RA, Ganote CE, Jennings RB (1974) The "no- reflow" phenomenon after temporary coronary occlusion in the dog. J Clin Invest 54: 1496-1506.

8. Fugit MD, Rubal BJ, Donovan DJ (2000) Effects of intracoronary nicardipine diltiazem, and verapamil on coronary blood flow. J Invasive Cardiol 12: 80-85.

9. Ndrepepa G, Tiroch K, Keta D (2010) Predictive Factors and Impact of No Reflow After Primary Percutaneous Coronary Intervention in Patients with Acute Myocardial Infarction. Circ Cardiovasc Interv 3: 27-33.

10. Iwakura K, Ito H, Ikushima M (2010) Association between hyperglycemia and the no-reflow phenomenon in patients with acute myocardial infarction. J Am Coll Cardiol 41: 1-7.

11. Niccoli G, Cosentino N, Spaziani C (2011) New strategies for the management of no-reflow after primary percutaneous coronary intervention. Expert Rev Cardiovasc Ther 9: 615-630.

12. Pantsios C, Kapelios C, Vakrou S (2016) Effect of Elevated Reperfusion Pressure on "No Reflow" Area and Infarct Size in a Porcine Model of IschemiaReperfusion. J Cardiovasc Pharmacol Ther 21: 405-411.

13. Forman MB, Jackson EK (2007) Importance of tissue perfusion in ST segment elevation myocardial infarction patients undergoing reperfusion strategies: role of adenosine. Clin Cardiol 30: 583-585.

14. Forman MB, Stone GW, Jackson EK (2006) Role of adenosine as adjunctive therapy in acute myocardial infarction. Cardiovasc Drug Rev 24: 116-147.

15. Forman MB, Hou D, Jackson EK (2008) Treating Acute "No-Reflow" with Intracoronary Adenosine. Tex Heart Inst J 35: 439-446.

16. Nazir SA, Khan JN, Mahmoud IZ (2014) The REFLO-STEMI trial comparing intracoronary adenosine, sodium nitroprusside and standard therapy for the attenuation of infarct size and microvascular obstruction during primary percutaneous coronary intervention: study protocol for a randomized controlled trial. Trials 15: p371.

17. Parham WA, Bouhasin A, Ciaramita JP (2004) Coronary hyperemic dose responses of intracoronary sodium nitroprusside. Circulation 109: 1236-1243.

18. Su Q, Li L, Naing KA, Sun Y (2014) Safety and effectiveness of nitroprusside in preventing no-reflow during percutaneous coronary intervention: a systematic review. Cell Biochem Biophys 68: 201-206.

19. Zhao S, Qi G, Tian W, Chen L, Sun Y (2014) Effect of intracoronary nitroprusside in preventing no reflow phenomenon during primary percutaneous coronary intervention: a meta-analysis. J Interv Cardiol 27: 356-364.

20. Wang L, Cheng Z, Gu Y, Peng D (2015) Short-term effects of verapamil and diltiazem in the treatment of no reflow phenomenon: a meta-analysis of randomized controlled trials. Biomed Res Int 2015: p382086.

21. Huang R, Patel P, Walinsky $P$ (2006) Efficacy of intracoronary nicardipine in the treatment of no-reflow during PCl. Catheter Cardiovasc Interv 68: 671-676.

22. Niccoli G, Spaziani C, Marino M (2010) Effect of chronic aspirin therapy on angiographic thrombus burden in patients admitted for a first ST-elevation myocardial infarction. Am J Cardiol 105: 587-591.

23. Mangiacapra F, Muller O, Ntalianis A (2010) Comparison of 600 versus $300-\mathrm{mg}$ clopidogrel loading dose in patients with STEMI undergoing primary coronary angioplasty. Am J Cardiol 106: 1208-1211.

24. Gibson CM, de Lemos JA, Murphy SA (2001) Combination therapy with abciximab reduces angiographically evident thrombus in acute myocardial infarction: a TIMI 14 substudy. Circulation 103: 2550-2554.

25. Piot $C$, Croisille $P$, Staat $P$ (2008) Effect of cyclosporine on reperfusion injury in acute myocardial infarction. N Engl J Med 359: 473-481.

26. Atar D, Petzelbauer P, Schwitter J (2009) for the F.I.R.E. Investigators Effect of intra- venous FX06 as an adjunct to primary percutaneous coronary intervention for acute ST-segment elevation myocardial infarction results of the F.I.R.E. (Efficacy of FX06 in the Prevention of Myocardial Reperfusion Injury) trial. J Am Coll Cardiol 53: 720-729.

27. Armstrong PW, Granger CB, Adams PX (2007) APEX AMI Investigators. Pexelizumab for acute ST-elevation myocardial infarction in patients undergoing primary percutaneous coronary intervention: a randomized controlled trial. JAMA 297: 43-51. 
Citation: Karimianpour A, Maran A (2017) Impervious Coronary No-Reflow: A Case Study. Cardiovasc Pharm Open Access 6: 229. doi: 10.4172/23296607.1000229

28. Hale SL, Kloner RA (2015) Dabigatran treatment: effects on infarct size and the no-reflow phenomenon in a model of acute myocardial ischemia/reperfusion. $J$ Thromb Thrombolysis 39: 50-544.

29. Chen WR, Tian F, Chen YD (2016) Effects of liraglutide on no-reflow in patients with acute ST-segment elevation myocardial infarction. Inter J Cardiol 208: 109-114.
30. Parsa CJ, Matsumoto A, Kim J, Riel RU (2003) A novel protective effect of erythropoietin in the infarcted heart. J Clin Invest 112: 999-1007.

31. Falase B, Easaw J, Youhana A (2001) The role of nicorandil in the treatment of myocardial ischaemia. Expert Opin Pharmacother 2: 845-856.

32. Iwakura J, Ito $\mathrm{H}$, Okamura A (2009) Nicorandil treatment in patients with acute myocardial infarction: a meta-analysis. Circ J 73: 925-931. 